\title{
Research on the Function, the Influencing Factors and Implementation Strategies of Family Sports
}

\author{
Qingbao Wang \\ College of Physical Education/Family Education Research Center, Baicheng Normal University \\ Baicheng, Jilin, China 137000
}

\begin{abstract}
Under the background that national fitness has been increasingly accepted by vast citizens as the national strategy to realize the Chinese Dream, family sports have also obtained unprecedented attentions as one of the most effective approaches to promote lifetime sports. This research carries out the analysis on the main influencing factors based on current function of family sports, puts forward countermeasures beneficial to the implementation and continuous development of family sports in our country, and then improves family sports awareness and value concept of parents and teenagers, encourages more family members to be engaged in family sports activities, and promotes the comprehensive development of family education.
\end{abstract}

Keywords-family sports; function: influencing factors; implementation strategies; family education

\section{INTRODUCTION}

Over the years, our country has shown fast economic development, and constantly improved residents' living standard, and sports activities have been accepted and favored by more and more families and individuals, become inseparable contents and components of family life, and are the main approach to promote harmonious families. Sports activities among individuals of family members can not only improve the physical and psychological health of family members, but also enrich family life, and benefit social stability and harmonious development, while promoting the harmonious relationship of families. Clear out and further expand relevant concepts of family sports from the perspective of national fitness and lifetime sports, in respect of the connotation and extension in the new era, actively expand the interaction and influencing factors of family education, school sports and social sports, promote mutual coordination and promote the comprehensive development of family education.

\section{FUNCTION AND EFFECT OF FAMILY SPORTS}

\section{A. Family sports is an important component of quality education}

Quality education emphasizes national publicity, and requires students to develop morally, intellectually and physically, and further develops the cultural, psychological and physical quality of teenagers. However, physical exercise is one of the most effective approaches to get a good physical fitness and psychological quality. Teenagers are the most active participators in family sports, and by carrying out benign family sports, it can not only reinforce physical and psychological health, but also help parents adopt corresponding education measures and approaches by physical exercise based on their children's sports activities. The natural instincts of children will be revealed unconsciously in physical exercise, brave or scared, smart or dull, so parents can understand and master children's personality characteristics in real time, and then guide and educate effectively, promote children to form a good psychological quality and sound mental structure under the environment of family physical exercise, and lay a solid foundation for comprehensive quality education.

\section{B. Family sports is precondition to carry out school sports and social sports}

Schools and families are two main environments in students' growth and personality cultivation, while family education is the starting point for people to receive all basic educations. Practices of family education and school education indicate that, the development of school education cannot be separated from the support and cooperation of family education, and similarly, school sports cannot be separated from family sports. Family sports are the continuity, extension and consolidation of school sports in time and space, and also the basis to carry out social sports. Teenagers require appropriate physical exercises and activities in different living environments and periods of time, to keep an excellent physical and mental state. As the organic combination of lifetime sports system, family sports, school sports and social sports are dispensable in the development of family sports and sports education, and family sports are the starting point and also ending point of lifetime sports, and will run though the whole course of lifetime sports.

\section{Family sports is an effective approach to build a harmonious society}

Harmonious society is a good society for which people have been seeking, and must adhere to "people-oriented", which is also the essential starting point and foothold to build a harmonious socialist society, and the harmonious relationship of family members as a part of the society plays a crucial role in building a harmonious society. Therefore, in the construction of harmonious society, the primary basic task is to build harmonious families. Family members take an active part in sports activities based on sports interests, and experience the interests brought by sports as equal social identity, without differences and discriminations in the social hierarchy, so it can exert a positive effect on the social development and the progress of individual members. 


\section{Family sports is an important link to inherit the value of cultural education}

Sports culture refers to the era characteristics, regional features and national styles of people reflected by sports activities in the process of changing the objective world, regulating personal emotions and coordinating group relationship. As a special cultural mode, it is an important component of social culture, and the development of sports culture is beneficial to the harmonious development and cultural progress of humans. Famous Educator, Mr. Xingzhi TAO used to point out that, "health is the starting point of life, and also the essential starting point for education". The growth of teenagers and children, and cannot be separated from family cultivation. As an important component of family education, family sports can exert an obvious effect on cultivating the physical exercise awareness and motivation of family members as well as the habit of lifetime sports.

\section{FACTORS INFLUENCING FAMILY SPORTS EFFECT}

\section{A. The development level of social economic}

The development of family sports cannot be separated from sports development, and the core of sports development is the continuous development of physical and psychological health, and depends on the continuous increase in the national economy. Economic factors are the essential power for social development, and economic development also depends on the improved overall quality of all citizens. Sports development can improve the national comprehensive quality, and the physical quality, and then promote the economic development level in a certain degree as well as sports development, especially the development of family sports. Therefore, as an important component of sports, family sports can be sustainably developed by relying on the continuous development of national economy. Besides, it can also provide power for economic development and supplement each other.

\section{B. Family environment factors}

Family is one of the most essential social units as well as the most basic and critical system and group form of humans, and also the existence of happy life. It can exert big influence on the ideology, behavior customs, and the formation of living attitude for member individuals, and is essential to the physical and psychological health of family members and also the foundation for member individuals to participate in physical exercise. As the basic unit of life, family environment can exert a direct influence on family sports, and parents' ideology, value orientation, occupation, hobbies, consumption psychology and their understanding and opinions about sports will directly influence their cultivation objective for their children as well as their children's interests and hobbies for sports.

\section{School environment factors}

School sports are sports activities focused on students at school, and by cultivating students' interests in sports, their attitude, habits, knowledge and capacity, it is aimed to reinforce students' physical fitness, cultivate students' moral and volitional quality, and promote students' physical and psychological health[5]. School sports are the basis for the physical exercise of teenagers, and family sports are also critical to the extension and continuity of school sports. Family members should accept systematic, sound and mandatory sports education at school in student times, during which school sports education is essential to cultivate lifetime sports. However, due to the restriction of school sports education in time and space, sports effect can be seriously affected, so we should encourage school sports education in families, and make families become the extension and continuity of school sports in time and space, and reinforce physical exercise effect.

\section{Mutual relation of school, society and family sports}

Family sports are spontaneously organized and participated by family members, with diversified modes, projects and sites and are sports activities mainly oriented to satisfy interests, hobbies, enrich family life and promote family stability by physical exercise activities[6]. As the enlightenment for the physical exercise of teenagers and infants, family sports are the basis for school sports, and also the important component of social sports. Young students receive systematic sports education at school, which will generate big influence on the cultivation of their lifetime sports concept, the habits of physical exercise, and the proficiency in sports skills, and lay a foundation for the development of family sports. Meanwhile, social sports have provided family members with convenient places, facilities and opportunities for physical exercise. Therefore, family sports, school sports and social sports are mutually influenced and supplemented.

\section{IMPLEMENTATION COUNTERMEASURES TO REINFORCE THE EFFECT OF FAMILY SPORTS}

\section{A. Accelerate economic development and promote material basis for family sports.}

The concept and behavior of family sports are related to the actual economic conditions and consumption concepts of families, and the development of family sports is not an isolated process, but relies on economics to some extent. As the awareness of family sports has become popular, family members have increased their understanding of sports, and taken an active part in all kinds of suitable physical exercises, to benefit the physical and psychological health of family members, a green life and open personality, and this has accelerated the development of family sports in our country. However, due to historical reasons and the national conditions, the overall level of our national economic development still has certain gap with developed countries. Besides, in respect of our weak foundation and more population, all of them have become major bottlenecks influencing the development of family sports in our country. Therefore, as long as we can accelerate our economic development, improve national living standard, and provide certain economic support to carry out and continue family sports, and then drive the consumption of family sports, and promote the development of family sports.

\section{B. Give full play to the spiritual and practical effect of families sports.}

Give fully play to family sports education, establish correct family sports concept, build a healthy family sports environment, and correctly guide teenagers to form a reasonable family sports behavior. Reasonably arrange physical exercise with suitable strength and scientific projects according to the characteristics and demands of teenagers' 
physical and mental development. Use the effective time and energy to lead children to participate in sports activities organized by the school and the society, lead them to actively apply the sports knowledge and skills learned in school and family sports education, dig out sports potential, form excellent sports habits, and make children experience hardship, sweating and joy in sports, learn about toughness and braveness and create a healthy body in sports, and work hard to become a natural person "who is smart, rational, and can handle affairs and run" from the perspective of Suo LU.

\section{Reinforce the promoting effect of school sports.}

There is no doubt that school sports can exert basic and promotional effect on family sports, so in order to better develop family sports, we should further optimize the reform of school sports education, and establish correct school and family sports concepts for vast students by improving the teaching technologies, skills, methods and concepts of PE teachers. Besides, by effectively linking sports class teaching with out-of-class and social sports activities, it can fully mobilize the enthusiasm for young students to participate in family, school and social sports activities. Ensure the effect of physical exercise by setting reasonable contents, forms and intensity of family sports homework, keep a close contact with parents to provide active support to students to join in-class and out-of-class sports activities, and give full play to the promotional effect of school sports.

\section{Coordinate the development of family, school and social sports.}

With respect to the essence of school sports, family sports and social sports, it is always "people-oriented", and the essential objective and tenet is to reinforce physical and psychological health, promote people to develop in an all-round way, and can reflect people's physical and psychological health, realize life value. These three items are mutually supplemented, and should be developed in a coordinated and balanced way. As we analyzes from the perspective of family sports, we can use the butterfly effect of family sports, to promote school sports teaching and social sports exercise. In the meantime, family sports can also promote emotional exchange of family members, form a harmonious and happy family atmosphere and lay a foundation to create a harmonious and stable society. Meanwhile, school sports and social sports have also provided technical, education and development support, in respect of the foundation of family sports, the guidance and promotion, etc. Therefore, we can not only strengthen the teaching achievements of school sports, and promote the quality of social sports development, but also promote the further development of family sports by combining family sports, school sports and social sports.

\section{V.CONCLUSION}

As an important mode to reinforce the family relationship as well as the physical and psychological health, the society, school and family members should attach importance to family sports. In light of its important role in social development and progress, we should reinforce the recognition of the family sports value and the discussion on the sustainable development of family sports, and provide support for the comprehensive development of family education.

\section{ACKNOWLEDGMENT}

Fund Project: Research on General Planning Topics by the Family Education Research Center of Baicheng Normal University; "13th Five Year Plan" Social Science Research Plan Topics of The Educational Department of Jilin Province.

\section{REFERENCES}

[1] Xiaojin LI and Junhui FENG. Research on the Positioning, Function and Development Strategies of Family Sports under the Perspective of Harmonious Society[J]. Sports Culture Guide, 2017,9(9): 40-44.

[2] Yuanchuan ZHANG. Discussion about the Formulation and Implementation of Family Sports Strategies[J]. BioTech World, 2013(2) 137-139.

[3] Xia DING, and Wei PENG. Research on the Current Status and Countermeasures of Family Sports Activities[J]. Journal of Heihe University, 2017,7(7): 46-47.

[4] Changqiao WU. Brief Discussion about the Effect of Developing Family Sports in Building a Harmonious Society[J]. Journal of Chifeng University (Natural Science Edition), 2017,3(3): 144-146.

[5] Youyu DU. Development of Vietnam Public Fitness Sports Club[D]. Beijing Sport University, 2017,6.

[6] Hao ZHANG. Source of Problems Influencing the Physical Health of Teenagers and the Solutions[J]. Journal of Longdong University, 2018,9 (5). : 88-92. 